\title{
Fluid - solid Coupling Analysis of Bioprosthetic Heart Valve Based on PISO Algorithm
}

\author{
Zhu Hongwei ${ }^{1, a}$, Yuan Quan ${ }^{1, b, *}$, Shen Bingshen ${ }^{1, c}$, Wang Zhichao ${ }^{1}$, $\operatorname{Tang} \operatorname{Dan}^{1}$ \\ ${ }^{1}$ Key Laboratory of High-efficiency and Clean Mechanical Manufacture, Shandong University, \\ Ministry of Education,Jinan, 250061, China. \\ asdlwzhwei@126.com, byuanquan66@sdu.edu.cn
}

Keywords: Biomechanical modeling; Bioprosthetic heart valve; Pressure implicit with splitting of operators(PISO)

\begin{abstract}
In this paper, according to the PISO algorithm in Fluent, the biomechanical analysis of the bioprosthetic heart valve model was carried out, and the deformation of the bioprosthetic valve was obtained. The simulation results show that the deformation of the bioprosthetic valve mainly occurs in the free edge of each leaflet, and the deformation of the abdomen is small, and the free edge will appear curl during the valve opening. In this paper, the same environment was used to simulate the pulsatile flow in the bioprosthetic valve, and the same result was obtained. The simulation and experimental results show that this method can be used in the fluid-solid coupling analysis of bioprosthetic valves, and provides a reference for the further optimization of bioprosthetic heart valve.
\end{abstract}

\section{Introduction}

Heart valve disease is fatal to the patient. The main treatment of it is artificial heart valve replacement $^{[1]}$. In recent years, the study of artificial heart valves focused on hemodynamics. The purpose of it is to find the blood and leaflet coupling mechanism ${ }^{[2,3]}$. For the fluid-solid coupling, the researchers have proposed many solutions, such as the finite element method,a classical method. When the valve leaflets are coupled with the blood, the valve leaflets have large deformation, because of the small elastic modulus. Moreover, the valve leaflet deformation occurs in a cardiac cycle in a very short period of time, so it can be applied to the use of ALE method for analysis ${ }^{[4]}$. PISO method is used to solve the pressure-velocity relation in the unsteady compressible or incompressible flow field, and the accuracy of the two-step correction is high. Therefore, this paper will combine ALE method and PISO algorithm to derive the valve leaflet, derive constitutive equation leaflets coupled with blood, and get the coupling speed iterative equation ${ }^{[5,6]}$. By the software of FLUENT, you can use the integrated PISO algorithm module leaflet deformation analysis.

\section{PISO algorithm}

In order to establish the mapping relationship between the coupling, PISO algorithm is used to discrete the momentum equation ${ }^{[7]}$. PISO is the improvement of SIMPLE algorithm. It uses a prediction step and two correction steps to calculate which could better meet the momentum equation and continuity equation. 
The discretization process is as follow:

(1) The step of prediction. Predicted pressure field is set as $p^{*}$. Then, the velocity components $\mathrm{u}^{*}$ and $v^{*}$ could be gotten by solving the discrete form of momentum equation.

$$
\begin{aligned}
& a_{i, J} u_{I, J}{ }^{*}=\sum a_{n b} u_{n b}^{*}+\left(p_{I-1, J}^{*}-p_{I, J}^{*}\right) A_{I, J}+b_{I, J} \\
& a_{I, J} v_{I, J}{ }^{*}=\sum a_{n b} v_{n b}^{*}+\left(p_{I, J-1}^{*}-p_{I, J}^{*}\right) A_{I, J}+b_{I, J}
\end{aligned}
$$

(2)The first step of modification. Velocity field, which is gotten by the predicted pressure field, generally doesn't satisfy the continuity equation. Therefore, the step of prediction is used, and it asked the velocity components $\mathrm{u}^{*}$ and $v^{*}$ to meet:

$$
\begin{aligned}
& p^{* *}=p^{*}+p^{\prime}, \\
& v^{* *}=v^{*}+v^{\prime}, \\
& u^{* *}=u^{*}+u^{\prime},
\end{aligned}
$$

The velocity components after the first step of modification are

$$
\begin{aligned}
& u_{i, j}^{* *}=u_{i, j}^{*}+d_{i, j}\left(p_{i-1, j}^{\prime}-p_{i, j}^{\prime}\right) \\
& v_{i, j}^{* *}=v_{i, j}^{*}+d_{i, j}\left(p_{i-1, j}^{\prime}-p_{i, j}^{\prime}\right)
\end{aligned}
$$

(3) The second step of modification. Momentum dispersion equations of $u^{* *}$ and $v^{* *}$ are:

$$
\begin{aligned}
& a_{i, j} u_{i, j}^{* *}=\sum a_{n b} u_{n b}^{*}+\left(p_{i-1, j}^{* *}-p_{i, j}^{* *}\right) A_{i, j}+b_{i, j}, \\
& a_{i, j} v_{i, j}^{* *}=\sum a_{n b} v_{n b}^{*}+\left(p_{i, j-1}^{* *}-p_{i, j}^{* *}\right) A_{i, j}+b_{i, j},
\end{aligned}
$$

According to the solution of momentum dispersion equations, $\mathrm{u}^{* * *}$ and $v^{* * *}$, after the second step of modification, are gotten:

$$
\begin{aligned}
& a_{i, j} u_{i, j}^{* * *}=\sum a_{n b} u_{n b}^{* *}+\left(p_{i-1, j}^{* * *}-p_{i, j}^{* * *}\right) A_{i, j}+b_{i, j}, \\
& a_{i, j} v_{i, j}^{* * *}=\sum a_{n b} v_{n b}^{* *}+\left(p_{i, j-1}^{* * *}-p_{i, j}^{* * *}\right) A_{i, j}+b_{i, j},
\end{aligned}
$$

If the equations aren't convergence, the next iteration need to be done.

\section{Model and the boundary condition}

In this paper, the classical ellipsoidal bioprosthetic valves ${ }^{[8]}$ were used, in which the leaflets and aortic sinuses of the heart valves were modeled separately, and then assembled in ANSYS.Carried out in the assembly model in ANSYS Workbench, and Boolean operations were used to remove the solid portions (leaflets) which were contained int he fluid portions (blood). Then, bioprosthetic valve model from the Fluent (see Fig. 1) was gotten.

In a cardiac cycle, the heart valve is constantly open and closed. The key to coupling blood and leaflet analysis is to analyze the valve opening or closing process, which is a very short process. When the aortic pressure is greater than the pressure of the ventricle, the valve opens; and when the ventricular pressure is greater than the aorta,the valve closes. In this paper, the following blood inlet velocity curve (see Fig. 2) was established to simulate cardiac pressure changes. 


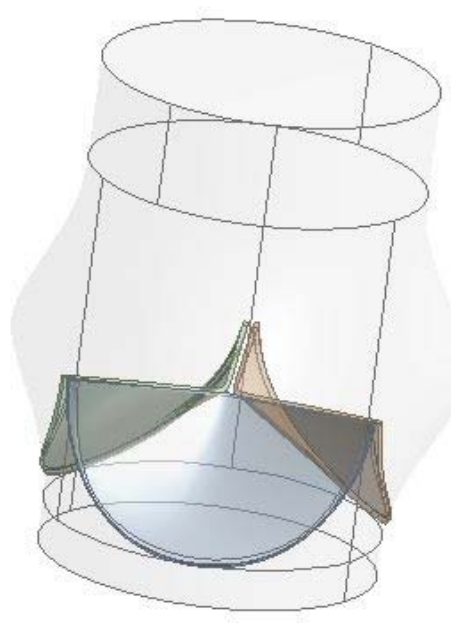

Fig. 1 Bioprosthetic valve model from the Fluent

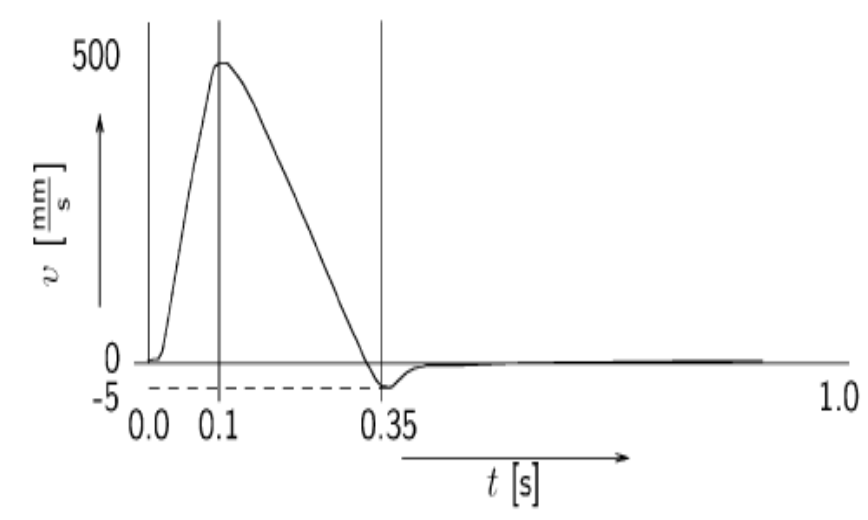

Fig. 2 The following blood inlet velocity curve

In addition to the entry speed, the other parameters are defined as follows:The blood was regarded as the uncompressed viscous fluid, the blood density was $1.105 \mathrm{~g} / \mathrm{cm} 3$, the blood viscosity was $0.00466 \mathrm{~Pa} \cdot \mathrm{s}$, the elastic modulus is $3 \times 108 \mathrm{~N} / \mathrm{m} 2$, the temperature was defined according to the human body normal temperature is $309.15 \mathrm{~K}^{[9]}$. Meanwhile, the density of the bioprosthetic valve was set to $1.2 \mathrm{~g} / \mathrm{cm} 3$, the Poisson's ratio was 0.3 , and the elastic modulus was set to $15 \mathrm{MPa}$.

In Fluent, one-way coupling can be achieved. This coupling method is to calculate the fluid (the blood in this article) flow field firstly, and then the results passed to the solid (bioprosthetic valve) for calculation. In this paper, Fluent module was used for simulation, and ANSYS WORKBENCH platform was used to build a framework for coupling calculation.

The parameters of the blood and the parameters of the bioprosthetic valve are set according to section 2.2. SIMPLE, SIMPLER and PISO algorithms are used to calculate the fluid fraction in the Fluent. In this paper, the same PISO algorithm as the theoretical partial discretization method is used to calculate the fluid fraction. The results are transferred to the structural part for leaflet analysis. And finally get the bioprosthetic valve of the deformation process, the process of interception of the valve open in the screenshot ( see Fig. 3). 

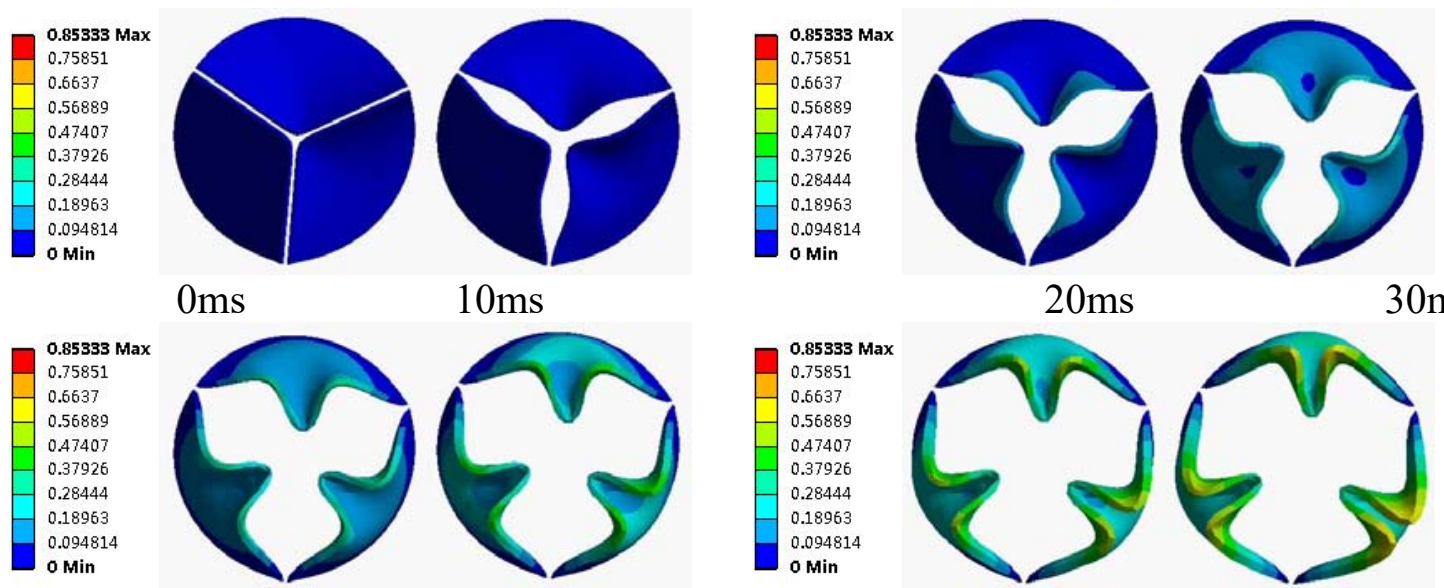

$10 \mathrm{~ms}$

$20 \mathrm{~ms}$

$40 \mathrm{~ms}$

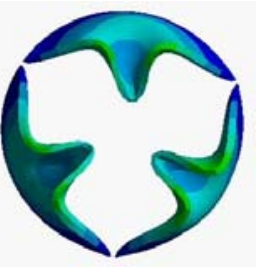

$50 \mathrm{~ms}$

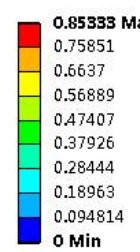

0.09481

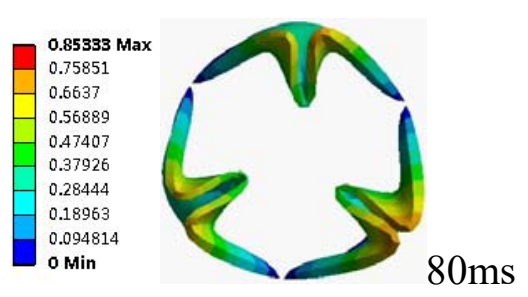

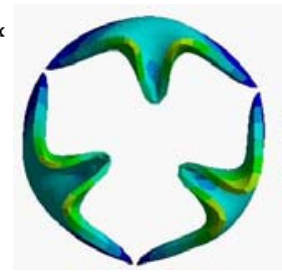

$60 \mathrm{~ms}$
$30 \mathrm{~ms}$

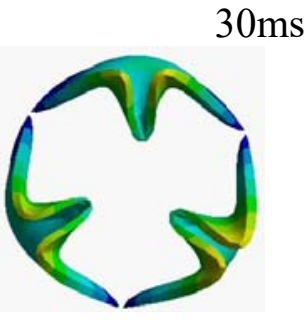

$70 \mathrm{~ms}$

Fig. 3 The Opening Process of Bioprosthetic Valve under Fluent Simulation

From the simulation results can be drawn: (1) In $0.8 \mathrm{~s}$, the bioprosthetic valve is gradually opened, the effective opening area gradually increased until the bioprosthetic valve is completely open; in the valve opening process, the valve leaflet deformation is mainly concentrated in the bioprosthetic valve valve leaf free edge; you can see the orange part of the deformation of the largest, in the valve leaflets and leaflets between the junction and the valve leaflets of the lower abdomen deformation. (2) In the process of valve leaf opening, the free edge of the valve leaf near the central part of the part of the curl; the valve is fully open after the opening area of the shape of the approximate triangular.

\section{Conclusions}

In this paper, the coupling equations of bioprosthetic valve and blood were deduced, and the momentum equation of blood was discretized. The velocity iteration equation of coupling process was obtained. Meanwhile, the three-dimensional model of bioprosthetic valve was established. The coupling process between bioprosthetic valve and blood was simulated by using PISO algorithm integrated in Fluent, and the deformation of bioprosthetic valve under blood impact was obtained.From the simulation results can be drawn: (1) The deformation of the bioprosthetic valve mainly occurs in the free edge of each leaflet, and the deformation of the abdomen is small. (2)the free edge will appear curl during the valve opening.

\section{Acknowledgements}

This work was financially supported by the National Natural Science Foundation of China (31170906).

\section{References}

[1] Li J, Luo X Y, Kuang Z B. A nonlinear anisotropic model for porcine aortic heart valves - Journal of 
Biomechanics[J]. Journal of Biomechanics, 34(10), (2001), p. 1279-89

[2] Marom G. Numerical Methods for Fluid-Structure Interaction Models of Aortic Valves[J]. Archives of Computational Methods in Engineering, 22(4), (2015), p. 1-26

[3] Tang D, Yang C, Geva T, et al. Image-Based Patient-Specific Ventricle Models with Fluid-Structure Interaction for Cardiac Function Assessment and Surgical Design Optimization.[J]. Progress in Pediatric Cardiology, 30(1-2),(2010), p. 51-62

[4] Zhang Aman. Fluid - solid coupling dynamics[M]. National Defense Industry Press, (2011)

[5] Nobili, M., U. Morbiducci, and R. Ponzini. "Numerical simulation of the dynamics of a bileaflet prosthetic heart valve using a fluid-structure interaction.

[6] Hart J D, Baaijens F P T, Peters G W M, et al. A computational fluid-structure interaction analysis of a fiber-reinforced stentless aortic valve.[J]. Journal of Biomechanics, 36(5), (2003), p. 699-712.

[7] WANG Weishu, XU Weihui, ZHAI Zhaojiang, et al. Realization of PISO Algorithm and Comparison Convergence Characteristics with SIMPLE Series Algorithms[J]. Journal of North China Institute of Water Conservancy and Hydroelectric Power,28(4), (2007), p. 33-36.

[8] Ma Xvejie, Du Yawei, Zhang Linan, et al. Fluid Solid Interaction Analysis of Bioprosthetic Heart Valve[J]. Chinese Journal of Medical Instrumentation,38(5),(2014), p. 325-328.

[9] Annerel S, Degroote J, Claessens T, et al. The upstream boundary condition influences the leaflet opening dynamics in the numerical FSI simulation of an aortic BMHV[J]. International Journal for Numerical Methods in Biomedical Engineering,28(6-7), (2012), p. 745-760. 